\title{
Fuzzy fixed point theorems in Hausdorff fuzzy metric spaces
}

Supak Phiangsungnoen ${ }^{1}$, Wutiphol Sintunavarat ${ }^{2 *}$ and Poom Kumam ${ }^{1 *}$

${ }^{*}$ Correspondence:

wutiphol@mathstat.sci.tu.ac.th; poom_teun@hotmail.com; poom.kum@kmutt.ac.th

${ }^{1}$ Department of Mathematics, Faculty of Science, King Mongkut's University of Technology Thonburi (KMUTT), 126 Pracha Uthit Rd., Bang Mod, Thung Khru, Bangkok, 10140, Thailand

${ }^{2}$ Department of Mathematics and Statistics, Faculty of Science and Technology, Thammasat University Rangsit Center, Pathumthani, 12121, Thailand

\begin{abstract}
In this paper, we introduce the concept of fuzzy mappings in Hausdorff fuzzy metric spaces (in the sense of George and Veeramani (Fuzzy Sets Syst. 64:395-399, 1994)). We establish the existence of $\alpha$-fuzzy fixed point theorems for fuzzy mappings in Hausdorff fuzzy metric spaces, which can be utilized to derive fixed point theorems for multivalued mappings. We also give an illustrative example to support our main result.
\end{abstract}

Keywords: $\alpha$-fuzzy fixed point; fuzzy mappings; fuzzy metric spaces; Hausdorff fuzzy metric spaces

\section{Introduction}

In 1965, Zadeh [1] introduced and studied the concept of a fuzzy set in his seminal paper. Afterward, several researches have extensively developed the concept of fuzzy set, which also include interesting applications of this theory in different fields such as mathematical programming, modeling theory, control theory, neural network theory, stability theory, engineering sciences, medical sciences, color image processing, etc. The concept of fuzzy metric spaces was introduced initially by Kramosil and Michalek [2]. Later on, George and Veeramani [3] modified the notion of fuzzy metric spaces due to Kramosil and Michalek [2] and studied a Hausdorff topology of fuzzy metric spaces. Recently, Gregori et al. [4] gave many interesting examples of fuzzy metrics in the sense of George and Veeramani [3] and have also applied these fuzzy metrics to color image processing. Several researchers proved the fixed point theorems in fuzzy metric spaces such as in [5-20] and the references therein. In 2004, López and Romaguera [21] introduced the Hausdorff fuzzy metric on a collection of nonempty compact subsets of a given fuzzy metric spaces. Recently, Kiany and Amini-Harandi [22] proved fixed point and endpoint theorems for multivalued contraction mappings in fuzzy metric spaces.

On the other hand, Heilpern [23] first introduced the concept of fuzzy contraction mappings and proved a fixed point theorem for fuzzy contraction mappings in a complete metric linear spaces, which seems to be the first to establish a fuzzy analog of Nadler's contraction principle [24]. His work opened an avenue for further development of fixed point in this direction. Many researchers used different assumptions on various kinds of fuzzy mappings and proved several fuzzy fixed point theorems (see [25-35]) and references therein.

@2014 Phiangsungnoen et al.; licensee Springer. This is an Open Access article distributed under the terms of the Creative Commons Attribution License (http://creativecommons.org/licenses/by/2.0), which permits unrestricted use, distribution, and reproduction in any medium, provided the original work is properly cited. 
To the best of our knowledge, there is no discussion so far concerning the fuzzy fixed point theorems for fuzzy mappings in Hausdorff fuzzy metric spaces. The object of this paper is to study the role of some type of fuzzy mappings to ascertain the existence of fuzzy fixed point in Hausdorff fuzzy metric spaces. We also present some relation of multivalued mappings and fuzzy mappings.

\section{Preliminaries}

Firstly, we recall some definitions and properties of an $\alpha$-fuzzy fixed point.

Let $X$ be an arbitrary nonempty set. A fuzzy set in $X$ is a function with domain $X$ and values in [0,1]. If $A$ is a fuzzy set and $x \in X$, then the function-value $A(x)$ is called the grade of membership of $x$ in $A . \mathcal{F}(X)$ stands for the collection of all fuzzy sets in $X$ unless and until stated otherwise.

Definition 2.1 Let $X$ and $Y$ be two arbitrary nonempty sets. A mapping $T$ from the set $X$ into $\mathcal{F}(Y)$ is said to be a fuzzy mapping.

If $X$ is endowed with a topology, for $\alpha \in[0,1]$, the $\alpha$-level set of $A$ is denoted by $[A]_{\alpha}$ and is defined as follows:

$$
[A]_{\alpha}=\{x \in X: A(x) \geq \alpha\} ; \quad \alpha \in(0,1]
$$

and

$$
[A]_{0}=\overline{\{x \in X: A(x)>0\}},
$$

where $\bar{B}$ denotes the closure of $B$ in $X$.

Definition 2.2 Let $X$ be an arbitrary nonempty set, $T$ be fuzzy mapping from $X$ into $\mathcal{F}(X)$ and $z \in X$. If there exists $\alpha \in[0,1]$ such that $z \in[T z]_{\alpha}$, then a point $z$ is called an $\alpha$-fuzzy fixed point of $T$.

The following notations as regards $t$-norm and fuzzy metric space will be used in the sequel.

Definition 2.3 ([36]) A binary operation $*:[0,1]^{2} \rightarrow[0,1]$ is a continuous $t$-norm if it satisfies the following conditions:

(T1) $*$ is associative and commutative,

(T2) $*$ is continuous,

(T3) $a * 1=a$ for all $a \in[0,1]$,

(T4) $a * b \leq c * d$ whenever $a \leq c$ and $b \leq d$ for all $a, b, c, d \in[0,1]$.

Examples of a continuous $t$-norm are Lukasievicz $t$-norm, that is, $a *_{L} b=\max \{a+b-$ $1,0\}$, product $t$-norm, that is, $a *_{P} b=a b$ and minimum $t$-norm, that is, $a *_{M} b=\min \{a, b\}$.

The concept of fuzzy metric space is defined by George and Veeramani [3] as follows.

Definition 2.4 ([3]) Let $X$ be an arbitrary nonempty set, $*$ be a continuous $t$-norm, and $M$ be a fuzzy set on $X^{2} \times(0, \infty)$. The 3 -tuple $(X, M, *)$ is called a fuzzy metric space if satisfying the following conditions, for each $x, y, z \in X$ and $t, s>0$, 
(M1) $M(x, y, t)>0$,

(M2) $M(x, y, t)=1$ if and only if $x=y$,

(M3) $M(x, y, t)=M(y, x, t)$,

(M4) $M(x, y, t) * M(y, z, s) \leq M(x, z, t+s)$,

(M5) $M(x, y, \cdot):(0, \infty) \rightarrow[0,1]$ is continuous.

Remark 2.5 It is worth pointing out that $0<M(x, y, t)<1$ (for all $t>0$ ) provided $x \neq y$ (see [37]).

Let $(X, M, *)$ be a fuzzy metric space. For $t>0$, the open ball $B(x, r, t)$ with a center $x \in X$ and a radius $0<r<1$ is defined by

$$
B(x, r, t)=\{y \in X: M(x, y, t)>1-r\} .
$$

A subset $A \subset X$ is called open if for each $x \in A$, there exist $t>0$ and $0<r<1$ such that $B(x, r, t) \subset A$. Let $\tau$ denote the family of all open subsets of $X$. Then $\tau$ is a topology on $X$, called the topology induced by the fuzzy metric $M$. This topology is metrizable (see [38]).

Example 2.6 ([3]) Let $(X, d)$ be a metric space. Define $a * b=a b$ (or $a * b=\min \{a, b\})$ for all $a, b \in[0,1]$, and define $M: X^{2} \times(0, \infty) \rightarrow[0,1]$ as

$$
M(x, y, t)=\frac{t}{t+d(x, y)}
$$

for all $x, y \in X$ and $t>0$. Then $(X, M, *)$ is a fuzzy metric space. We call this fuzzy metric induced by the metric $d$ the standard fuzzy metric.

Now we give some examples of fuzzy metric space due to Gregori et al. [4].

Example 2.7 ([4]) Let $X$ be a nonempty set, $f: X \rightarrow \mathbb{R}^{+}$be a one-one function and $g$ : $\mathbb{R}^{+} \rightarrow[0, \infty)$ be an increasing continuous function. For fixed $\alpha, \beta>0$, define $M: X^{2} \times$ $(0, \infty) \rightarrow[0,1]$ as

$$
M(x, y, t)=\left(\frac{(\min \{f(x), f(y)\})^{\alpha}+g(t)}{(\max \{f(x), f(y)\})^{\alpha}+g(t)}\right)^{\beta}
$$

for all $x, y \in X$ and $t>0$. Then $(X, M, *)$ is a fuzzy metric space on $X$ where $*$ is the product $t$-norm.

Example 2.8 ([4]) Let $(X, d)$ be a metric space and $g: \mathbb{R}^{+} \rightarrow[0, \infty)$ be an increasing continuous function. Define $M: X^{2} \times(0, \infty) \rightarrow[0,1]$ as

$$
M(x, y, t)=e^{\left(-\frac{d(x, y)}{g(t)}\right)}
$$

for all $x, y \in X$ and $t>0$. Then $(X, M, *)$ is a fuzzy metric space on $X$ where $*$ is the product $t$-norm. 
Example 2.9 ([4]) Let $(X, d)$ be a bounded metric space with $d(x, y)<k$ (for all $x, y \in X$, where $k$ is fixed constant in $(0, \infty))$ and $g: \mathbb{R}^{+} \rightarrow(k, \infty)$ be an increasing continuous function. Define a function $M: X^{2} \times(0, \infty) \rightarrow[0,1]$ as

$$
M(x, y, t)=1-\frac{d(x, y)}{g(t)}
$$

for all $x, y \in X$ and $t>0$. Then $(X, M, *)$ is a fuzzy metric space on $X$ where $*$ is a Lukasievicz $t$-norm.

Definition $2.10([3])$ Let $(X, M, *)$ be a fuzzy metric space.

(1) A sequence $\left\{x_{n}\right\}$ in $X$ is said to be convergent to a point $x \in X$ if $\lim _{n \rightarrow \infty} M\left(x_{n}, x, t\right)=1$ for all $t>0$.

(2) A sequence $\left\{x_{n}\right\}$ in $X$ is called a Cauchy sequence if, for each $0<\epsilon<1$ and $t>0$, there exists $n_{0} \in \mathbb{N}$ such that $M\left(x_{n}, x_{m}, t\right)>1-\epsilon$ for each $n, m \geq n_{0}$.

(3) A fuzzy metric space in which every Cauchy sequence is convergent is said to be complete.

(4) A fuzzy metric space in which every sequence has a convergent subsequence is said to be compact.

Lemma $2.11([6])$ Let $(X, M, *)$ be a fuzzy metric space. For all $x, y \in X, M(x, y, \cdot)$ is nondecreasing function.

If $(X, M, *)$ is a fuzzy metric space, then the mapping $M$ is continuous on $X^{2} \times(0, \infty)$, that is, if $\left\{x_{n}\right\},\left\{y_{n}\right\} \subseteq X$ are sequences such that $\left\{x_{n}\right\} \stackrel{M}{\rightarrow} x \in X,\left\{y_{n}\right\} \stackrel{M}{\rightarrow} y \in X$ and $\left\{t_{n}\right\} \subset$ $(0, \infty)$ verifies $\left\{t_{n}\right\} \rightarrow t \in(0, \infty)$ then $\left\{M\left(x_{n}, y_{n}, t_{n}\right)\right\} \rightarrow M(x, y, t)$.

Lemma 2.12 ([21]) If $(X, M, *)$ be a fuzzy metric space, then $M$ is a continuous function on $X^{2} \times(0, \infty)$.

In 2004, Rodriguez-López and Romaguera [21] introduced the notion for Hausdorff fuzzy metric of a given fuzzy metric space $(X, M, *)$ on $\mathcal{K}_{M}(X)$, where $\mathcal{K}_{M}(X)$ denotes the set of its nonempty compact subsets.

Definition $2.13([21])$ Let $(X, M, *)$ be a fuzzy metric space. The Hausdorff fuzzy metric $H_{M}:\left(\mathcal{K}_{M}(X)\right)^{2} \times(0, \infty)$ is defined by

$$
H_{M}(A, B, t):=\min \left\{\inf _{x \in A}\left(\sup _{y \in B} M(x, y, t)\right), \inf _{y \in B}\left(\sup _{x \in A} M(x, y, t)\right)\right\}
$$

for all $A, B \in \mathcal{K}_{M}(X)$ and $t>0$.

Lemma $2.14([21])$ Let $(X, M, *)$ be a fuzzy metric space. Then the 3-tuple $\left(\mathcal{K}_{M}(X), H_{M}, *\right)$ is a fuzzy metric space.

Lemma 2.15 ([21]) Let $(X, M, *)$ be a fuzzy metric space and $t>0$ be fixed. If $A$ and $B$ are nonempty compact subsets of $X$ and $x \in A$, then there exists a point $y \in B$ such that

$$
\sup _{b \in B} M(x, b, t)=M(x, y, t) .
$$




\section{Main result}

In this section, we establish the existence theorem of fuzzy fixed point for $\alpha$-fuzzy mapping in Hausdorff fuzzy metric and reduce our result to metric space. The following lemma is essential in proving our main result.

Lemma 3.1 Let $(X, M, *)$ be a fuzzy metric space and $\left\{x_{n}\right\}$ is a sequence in $X$ such that for all $n \in \mathbb{N}$,

$$
M\left(x_{n+1}, x_{n+2}, k t\right) \geq M\left(x_{n}, x_{n+1}, t\right),
$$

where $0<k<1$. Suppose that

$$
\lim _{n \rightarrow \infty} *_{i=n}^{\infty} M\left(x_{1}, x_{2}, t h^{i}\right)=1
$$

for all $t>0$ and $h>1$. Then $\left\{x_{n}\right\}$ is a Cauchy sequence.

Proof It follows proof similar to the proof of Lemma 1 of Kiany and Amini-Harandi [22]. Then, in order to avoid repetition, the details are omitted.

Now we are ready to prove our main result.

Theorem 3.2 Let $(X, M, *)$ be a complete fuzzy metric space and $\alpha: X \rightarrow(0,1]$ be a mapping such that $[T x]_{\alpha(x)}$ is a nonempty compact subset of $X$ for all $x \in X$. Suppose that $T: X \rightarrow \mathcal{F}(X)$ is a fuzzy mapping such that

$$
H_{M}\left([T x]_{\alpha(x)},[T y]_{\alpha(y)}, k t\right) \geq M(x, y, t)
$$

for all $t>0$, where $k \in(0,1)$. If there exist $x_{0} \in X$ and $x_{1} \in\left[T x_{0}\right]_{\alpha\left(x_{0}\right)}$ such that

$$
\lim _{n \rightarrow \infty} *_{i=n}^{\infty} M\left(x_{0}, x_{1}, t h^{i}\right)=1
$$

for all $t>0$ and $h>1$, then $T$ has an $\alpha$-fuzzy fixed point.

Proof We start from $x_{0} \in X$ and $x_{1} \in\left[T x_{0}\right]_{\alpha\left(x_{0}\right)}$ under the hypothesis. From the assumption, we have $\left[T x_{1}\right]_{\alpha\left(x_{1}\right)}$ is a nonempty compact subset of $X$. If $\left[T x_{0}\right]_{\alpha\left(x_{0}\right)}=\left[T x_{1}\right]_{\alpha\left(x_{1}\right)}$, then $x_{1} \in\left[T x_{1}\right]_{\alpha\left(x_{1}\right)}$ and so $x_{1}$ is an $\alpha$-fuzzy fixed point of $T$ and the proof is finished. Therefore, we may assume that $\left[T x_{0}\right]_{\alpha\left(x_{0}\right)} \neq\left[T x_{1}\right]_{\alpha\left(x_{1}\right)}$. Since $x_{1} \in\left[T x_{0}\right]_{\alpha\left(x_{0}\right)}$ and $\left[T x_{1}\right]_{\alpha\left(x_{1}\right)}$ is a nonempty compact subset of $X$ then by Lemma 2.15 and condition (2), there exists $x_{2} \in\left[T x_{1}\right]_{\alpha\left(x_{1}\right)}$ satisfying

$$
\begin{aligned}
M\left(x_{1}, x_{2}, k t\right) & =\sup _{x_{2}^{\prime} \in\left[T x_{1}\right]_{\alpha\left(x_{1}\right)}} M\left(x_{1}, x_{2}^{\prime}, k t\right) \\
& \geq H_{M}\left(\left[T x_{0}\right]_{\alpha\left(x_{0}\right)},\left[T x_{1}\right]_{\alpha\left(x_{1}\right)}, k t\right) \\
& \geq M\left(x_{0}, x_{1}, t\right) .
\end{aligned}
$$

If $\left[T x_{1}\right]_{\alpha\left(x_{1}\right)}=\left[T x_{2}\right]_{\alpha\left(x_{2}\right)}$, then $x_{2} \in\left[T x_{2}\right]_{\alpha\left(x_{2}\right)}$. This implies that $x_{2}$ is an $\alpha$-fuzzy fixed point of $T$ and then the proof is finished. Therefore, we may assume that $\left[T x_{1}\right]_{\alpha\left(x_{1}\right)} \neq\left[T x_{2}\right]_{\alpha\left(x_{2}\right)}$. 
Since $x_{2} \in\left[T x_{1}\right]_{\alpha\left(x_{1}\right)}$ and $\left[T x_{2}\right]_{\alpha\left(x_{2}\right)}$ is a nonempty compact subset of $X$, by using Lemma 2.15 and condition (2), there exists $x_{3} \in\left[T x_{2}\right]_{\alpha\left(x_{2}\right)}$ satisfying

$$
\begin{aligned}
M\left(x_{2}, x_{3}, k t\right) & =\sup _{x_{3}^{\prime} \in\left[T x_{2}\right]_{\alpha\left(x_{2}\right)}} M\left(x_{2}, x_{3}^{\prime}, k t\right) \\
& \geq H_{M}\left(\left[T x_{1}\right]_{\alpha\left(x_{1}\right)},\left[T x_{2}\right]_{\alpha\left(x_{2}\right)}, k t\right) \\
& \geq M\left(x_{1}, x_{2}, t\right) .
\end{aligned}
$$

By induction, we can construct the sequence $\left\{x_{n}\right\}$ in $X$ such that $x_{n} \in\left[T x_{n-1}\right]_{\alpha\left(x_{n-1}\right)}$ and

$$
M\left(x_{n}, x_{n+1}, k t\right) \geq M\left(x_{n-1}, x_{n}, t\right)
$$

for all $n \in \mathbb{N}$. From Lemma 3.1, we get $\left\{x_{n}\right\}$ is a Cauchy sequence. Since $(X, M, *)$ is a complete fuzzy metric space, there exists $x \in X$ such that $\lim _{n \rightarrow \infty} x_{n}=x$, which means $\lim _{n \rightarrow \infty} M\left(x_{n}, x, t\right)=1$, for each $t>0$.

Now we claim that $x \in[T x]_{\alpha(x)}$. Since

$$
H_{M}\left(\left[T x_{n}\right]_{\alpha\left(x_{n}\right)},[T x]_{\alpha(x)}, k t\right) \geq M\left(x_{n}, x, t\right)
$$

and $\lim _{n \rightarrow \infty} M\left(x_{n}, x, t\right)=1$, then for each $t>0$, we get

$$
\lim _{n \rightarrow \infty} H_{M}\left(\left[T x_{n}\right]_{\alpha\left(x_{n}\right)},[T x]_{\alpha(x)}, k t\right)=1
$$

This implies that

$$
\lim _{n \rightarrow \infty} \sup _{x^{\prime} \in[T x]_{\alpha}(x)} M\left(x_{n+1}, x^{\prime}, t\right)=1
$$

and thus there exists a sequence $\left\{x_{n}^{\prime}\right\}$ in $[T x]_{\alpha(x)}$ such that

$$
\lim _{n \rightarrow \infty} M\left(x_{n}, x_{n}^{\prime}, t\right)=1,
$$

for each $t>0$. For each $n \in \mathbb{N}$, we have

$$
M\left(x_{n}^{\prime}, x, t\right) \geq M\left(x_{n}^{\prime}, x_{n}, \frac{t}{2}\right) * M\left(x_{n}, x, \frac{t}{2}\right)
$$

Since $\lim _{n \rightarrow \infty} M\left(x_{n}^{\prime}, x_{n}, \frac{t}{2}\right)=1$ and $\lim _{n \rightarrow \infty} M\left(x_{n}, x, \frac{t}{2}\right)=1$, we get

$$
\lim _{n \rightarrow \infty} M\left(x_{n}^{\prime}, x, t\right)=1
$$

that is, $\lim _{n \rightarrow \infty} x_{n}^{\prime}=x$. It follows from $[T x]_{\alpha(x)}$ being a compact subset of $X$ and $x_{n}^{\prime} \in[T x]_{\alpha(x)}$ that $x \in[T x]_{\alpha(x)}$. Therefore, $x$ is an $\alpha$-fuzzy fixed point of $T$. This completes the proof.

Next, we apply Theorem 3.2 to $\alpha$-fuzzy fixed point theorems in metric space. Before we study the following results, we give the following notation. 
Let $(X, d)$ be a metric space and $\mathcal{K}(X)$ denote the collection of all nonempty compact subsets of $X$. For $A, B \in \mathcal{K}(X)$, we denote

$$
H(A, B):=\max \left\{\sup _{x \in A}\left(\inf _{y \in B} d(x, y)\right), \sup _{y \in B}\left(\inf _{x \in A} d(x, y)\right)\right\}
$$

The function $H$ is called the Hausdorff metric. Further, it is well known that $(\mathcal{K}(X), H)$ is a metric spaces.

Corollary 3.3 Let $(X, d)$ be a complete metric space and $\alpha: X \rightarrow(0,1]$ be a mapping such that $[T x]_{\alpha(x)}$ is a nonempty compact subset of $X$ for all $x \in X$. Suppose that $T: X \rightarrow \mathcal{F}(X)$ be a fuzzy mapping such that

$$
H\left([T x]_{\alpha(x)},[T y]_{\alpha(y)}\right) \leq k d(x, y)
$$

for all $t>0$, where $k \in(0,1)$. Then $T$ has an $\alpha$-fuzzy fixed point.

Proof Let $(X, M, *)$ be standard fuzzy metric space induced by the metric $d$ with $a * b=a b$. Now we show that the conditions of Theorem 3.2 are satisfied. Since $(X, d)$ is a complete metric space then $(X, M, *)$ is complete. It is easy to see that $(X, M, *)$ satisfies (3). From Proposition 3 in [21], for each nonempty compact subset of $X$, we have

$$
H_{M}(A, B, t)=\frac{t}{t+H(A, B)} .
$$

By the above equality, we have

$$
\begin{aligned}
H_{M}\left([T x]_{\alpha(x)},[T y]_{\alpha(y)}, k t\right) & =\frac{k t}{k t+H\left([T x]_{\alpha(x),}[T y]_{\alpha(y)}\right)} \\
& \geq \frac{k t}{k(t+d(x, y))} \\
& \geq \frac{t}{t+d(x, y)} \\
& =M(x, y, t),
\end{aligned}
$$

for each $t>0$ and each $x, y \in X$. Therefore, the conclusion follows from Theorem 3.2.

Next, we give an example to support the validity of our results.

Example 3.4 Let $X=\{1,2,3\}$ and define metric $d: X \times X \rightarrow \mathbb{R}$ by

$$
d(x, y)= \begin{cases}0, & x=y, \\ \frac{1}{2}, & x \neq y \text { and } x, y \in\{1,3\}, \\ 1, & x \neq y \text { and } x, y \in\{1,2\}, \\ \frac{6}{11}, & x \neq y \text { and } x, y \in\{2,3\}\end{cases}
$$


It is easy to see that $(X, d)$ is a complete metric space. Denote $a * b=a b($ or $a * b=\min \{a, b\})$ for all $a, b \in[0,1]$ and

$$
M(x, y, t)=\frac{t}{t+d(x, y)}
$$

for all $x, y \in X$ and $t>0$. Then we find that $(X, M, *)$ is a complete fuzzy metric space. Define the fuzzy mapping $T: X \rightarrow \mathcal{F}(X)$ by

$$
\begin{aligned}
& (T 1)(t)=(T 2)(t) \begin{cases}\frac{3}{4}, & t=1, \\
0, & t=2,3,\end{cases} \\
& (T 3)(t)= \begin{cases}0, & t=1,3, \\
\frac{3}{4}, & t=2 .\end{cases}
\end{aligned}
$$

Define $\alpha: X \rightarrow(0,1]$ by $\alpha(x)=\frac{3}{4}$ for all $x \in X$. Now we obtain

$$
[T x]_{\frac{3}{4}}= \begin{cases}\{1\}, & x=1,2 \\ \{2\}, & x=3 .\end{cases}
$$

For $x, y \in X$, we get

$$
H\left([T x]_{\frac{3}{4}},[T y]_{\frac{3}{4}}\right)= \begin{cases}0, & x=y, \\ H(\{1\},\{1\})=0, & x \neq y \text { and } x=1, y=2, \\ H(\{1\},\{2\})=1, & x \neq y \text { and } x \in\{1,2\}, y=3, \\ H(\{2\},\{1\})=1, & x \neq y \text { and } x=3, y \in\{1,2\} .\end{cases}
$$

By a simple calculation, we get

$$
H_{M}\left([T x]_{\alpha(x)},[T y]_{\alpha(y)}, k t\right) \geq M(x, y, t)
$$

for all $t>0$, where $k=\frac{1}{4}$. Therefore all conditions of Theorem 3.2 hold and thus we can claim the existence of a point $z \in X$ such that $z \in[T z]_{\alpha(z)}$, that is, we have an $\alpha$-fuzzy fixed point of $T$. Thus $z=1$ is an $\alpha$-fuzzy fixed point of $T$.

Remark 3.5 From Example 3.4, we have

$$
H\left([T 1]_{\frac{3}{4}},[T 3]_{\frac{3}{4}}\right)=H(\{1\},\{2\})=1>\frac{\alpha}{2}=\alpha d(1,3)
$$

or $H\left([T 2]_{\frac{3}{4}},[T 3]_{\frac{3}{4}}\right)=H(\{1\},\{2\})=1>\frac{6 \alpha}{11}=\alpha d(2,3)$ for all $\alpha \in[0,1)$. Therefore, Corollary 3.3 is not applicable to claim the existence of an $\alpha$-fuzzy fixed point of $T$.

Here, we study some relations of multivalued mappings and fuzzy mappings. Indeed, we indicate that Corollary 3.3 can be utilized to derive a fixed point for a multivalued mapping. 
Corollary 3.6 Let $(X, d)$ be a complete metric space and $G: X \rightarrow \mathcal{K}(X)$ be multivalued mapping such that for all $x, y \in X$, we have

$$
H(G x, G y) \leq k d(x, y), \quad \text { where } k \in(0,1) .
$$

Then there exists $v \in X$ such that $v \in G v$.

Proof Let $\alpha: X \rightarrow(0,1]$ be an arbitrary mapping and $T: X \rightarrow \mathcal{F}(X)$ be defined by

$$
(T x)(t)= \begin{cases}\alpha(x), & t \in G x, \\ 0, & t \notin G x .\end{cases}
$$

By a routine calculation, we obtain

$$
[T x]_{\alpha(x)}=\{t:(T x)(t) \geq \alpha(x)\}=G x .
$$

Now condition (7) becomes condition (5). Therefore, Corollary 3.3 can be applied to obtain $v \in X$ such that $v \in[T v]_{\alpha(v)}=G v$. This implies that the multivalued mapping $G$ has a fixed point. This completes the proof.

\section{Conclusions}

In the present work we introduced a new concept of fuzzy mappings in the Hausdorff fuzzy metric space on compact sets, which is a partial generalization of fuzzy contractive mappings in the sense of George and Veeramani. Also, we derived the existence of $\alpha$-fuzzy fixed point theorems for fuzzy mappings in the Hausdorff fuzzy metric space. Moreover, we reduced our result from fuzzy mappings in Hausdorff fuzzy metric spaces to fuzzy mappings in metric space.

Finally, we showed some relation of multivalued mappings and fuzzy mappings, which can be utilized to derive fixed point for multivalued mappings.

\section{Competing interests}

The authors declare that they have no competing interests.

\section{Authors' contributions}

All authors contributed equally to the writing of this paper. All authors read and approved the final manuscript.

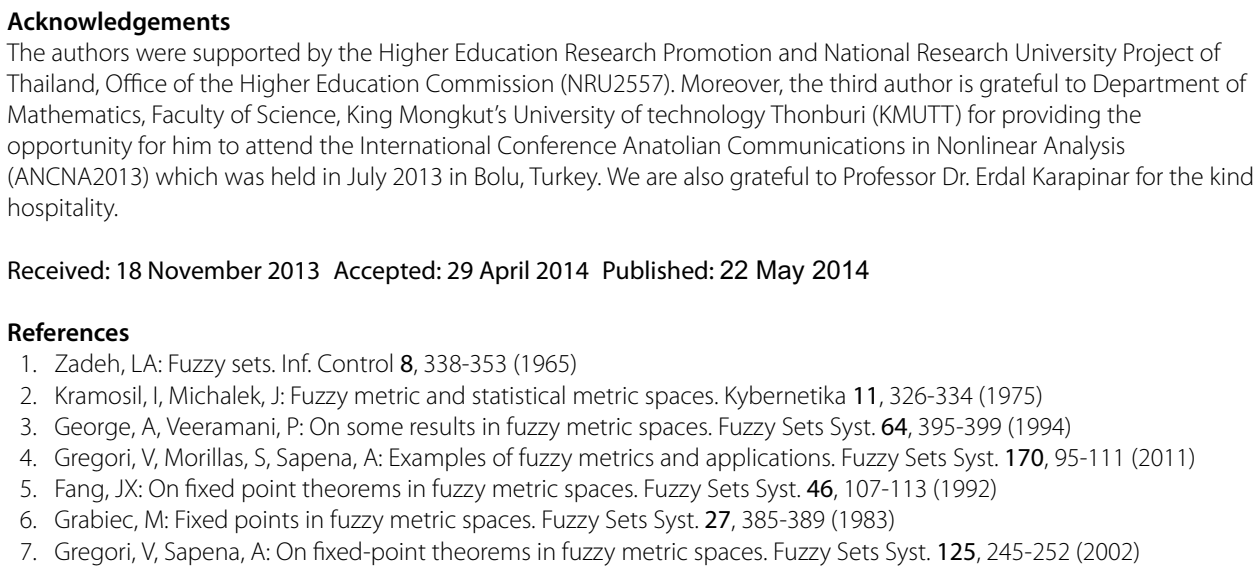


8. Miheţ, D: On the existence and the uniqueness of fixed points of Sehgal contractions. Fuzzy Sets Syst. 156, 135-141 (2005)

9. Miheț, D: On fuzzy contractive mappings in fuzzy metric spaces. Fuzzy Sets Syst. 158, 915-921 (2007)

10. Liu, Y, Li, Z: Coincidence point theorems in probabilistic and fuzzy metric spaces. Fuzzy Sets Syst. 158, 58-70 (2007)

11. Žikić, T: On fixed point theorems of Gregori and Sapena. Fuzzy Sets Syst. 144, 421-429 (2004)

12. Sintunavarat, W, Kumam, P: Common fixed point theorems for a pair of weakly compatible mappings in fuzzy metric spaces. J. Appl. Math. 2011, Article ID 637958 (2011)

13. Sintunavarat, W, Kumam, $P$ : Common fixed points for R-weakly commuting in fuzzy metric spaces. Ann. Univ. Ferrara 58, 389-406 (2012)

14. Chauhan, S, Sintunavarat, W, Kumam, P: Common fixed point theorems for weakly compatible mappings in fuzzy metric spaces using (JCLR) property. Appl. Math. 3(9), 976-982 (2012)

15. Chauhan, S, Alamgir Khan, M, Sintunavarat, W: Common fixed point theorems in fuzzy metric spaces satisfying $\phi$-contractive condition with common limit range property. Abstr. Appl. Anal. 2013, Article ID 735217 (2013)

16. Merghadi, F, Aliouche, A: A related fixed point theorem in $n$ fuzzy metric spaces. Iran. J. Fuzzy Syst. 7(3), 73-86 (2010)

17. Miheț, D: A class of contractions in fuzzy metric spaces. Fuzzy Sets Syst. 161, 1131-1137 (2010)

18. Roldán, A, Martínez-Moreno, J, Roldán, C, Cho, YJ: Multidimensional coincidence point results for compatible mappings in partially ordered fuzzy metric spaces. Fuzzy Sets Syst. (2013). doi:10.1016/j.fss.2013.10.009

19. Shen, YH, Qiu, D, Chen, W: Fixed point theory for cyclic $\varphi$-contractions in fuzzy metric spaces. Iran. J. Fuzzy Syst. 10(4), $125-133(2013)$

20. Xiao, J-Z, Zhu, X-H, Jin, X: Fixed point theorems for nonlinear contractions in Kaleva-Seikkala's type fuzzy metric spaces. Fuzzy Sets Syst. 200, 65-83 (2012)

21. Rodriguez-López, J, Romaguera, S: The Hausdorff fuzzy metric on compact sets. Fuzzy Sets Syst. 147, $273-283$ (2004)

22. Kiany, F, Amini-Harandi, A: Fixed point and endpoint theorems for set-valued fuzzy contraction maps in fuzzy metric spaces. Fixed Point Theory Appl. 2011, 94 (2011)

23. Heilpern, S: Fuzzy mappings and fixed point theorems. J. Math. Anal. Appl. 83, 566-569 (1981)

24. Nadler, SB Jr.: Multivalued contraction mapping. Pac. J. Math. 30(2), 475-488 (1969)

25. Estruch, VD, Vidal, A: A note on fixed fuzzy points for fuzzy mappings. Rend. Ist. Mat. Univ. Trieste 32, $39-45$ (2001)

26. Frigon, M, O'Regan, D: Fuzzy contractive maps and fuzzy fixed points. Fuzzy Sets Syst. 129, $39-45$ (2002)

27. Azam, A, Beg, l: Common fixed points of fuzzy maps. Math. Comput. Model. 49, 1331-1336 (2009)

28. Azam, A, Arshad, M, Beg, l: Fixed points of fuzzy contractive and fuzzy locally contractive maps. Chaos Solitons Fractals 42, 2836-2841 (2009)

29. Abbas, M, Damjanović, B, Lazović, R: Fuzzy common fixed point theorems for generalized contractive mappings. Appl. Math. Lett. 23(11), 1326-1330 (2010)

30. Bose, RK, Roychowdhury, MK: Fixed point theorems for generalized weakly contractive mappings. Surv. Math. Appl. 4 215-238 (2009)

31. Lee, BS, Cho, SJ: A fixed point theorem for contractive type fuzzy mappings. Fuzzy Sets Syst. 61, 309-312 (1994)

32. Rashwan, RA, Ahmed, MA: Common fixed point theorems for fuzzy mappings. Arch. Math. 38, 219-226 (2002)

33. Rhoades, BE: A common fixed point theorem for a sequence of fuzzy mappings. Int. J. Math. Sci. 18, 447-450 (1995)

34. Singh, B, Chauhan, MS: Fixed points of associated multimaps of fuzzy maps. Fuzzy Sets Syst. 110, 131-134 (2000)

35. Turkoglu, D, Rhoades, BE: A fixed fuzzy point for fuzzy mapping in complete metric spaces. Math. Commun. 10, 115-121 (2005)

36. Schweizer, B, Sklar, A: Statistical metric spaces. Pac. J. Math. 10, 313-334 (1960)

37. Mihet, D: Fixed point theorems in fuzzy metric spaces using property (E.A). Nonlinear Anal. 73(7), 2184-2188 (2010)

38. Gregori, V, Romaguera, S: Some properties of fuzzy metric spaces. Fuzzy Sets Syst. 115, 485-489 (2000)

10.1186/1029-242X-2014-201

Cite this article as: Phiangsungnoen et al.: Fuzzy fixed point theorems in Hausdorff fuzzy metric spaces. Journal of Inequalities and Applications 2014, 2014:201

\section{Submit your manuscript to a SpringerOpen ${ }^{\circ}$ journal and benefit from:}

- Convenient online submission

Rigorous peer review

- Immediate publication on acceptance

Open access: articles freely available online

- High visibility within the field

- Retaining the copyright to your article 